\title{
WNK Kinases, Renal Ion Transport and Hypertension
}

\author{
Pedro San-Cristobal $^{\mathrm{a}}$ Paola de los Heros ${ }^{\mathrm{a}}$ José Ponce-Coria ${ }^{\mathrm{a}}$ Erika Moreno ${ }^{\mathrm{a}} \mathrm{b}$ \\ Gerardo Gamba a \\ ${ }^{a}$ Molecular Physiology Unit, Instituto Nacional de Ciencias Médicas y Nutrición Salvador Zubirán and Instituto de \\ Investigaciones Biomédicas, Universidad Nacional Autónoma de México, Mexico City, and ${ }^{\text {b}}$ Universidad Autónoma \\ del Estado de Hidalgo, Pachuca, México
}

\section{Key Words}

Diuretics · Distal convoluted tubule $\cdot$ Salt transport •

Potassium excretion · Phosphorylation

\begin{abstract}
Two members of a recently discovered family of protein kinases are the cause of an inherited disease known as pseudohypoaldosteronism type II (PHAII). These patients exhibit arterial hypertension together with hyperkalemia and metabolic acidosis. This is a mirror image of Gitelman disease that is due to inactivating mutations of the SLC12A3 gene that encodes the thiazide-sensitive $\mathrm{Na}^{+}: \mathrm{Cl}^{-}$cotransporter. The uncovered genes causing PHAll encode for serine/threonine kinases known as WNK1 and WNK4. Physiological and biochemical studies have revealed that WNK1 and WNK4 modulate the activity of several transport pathways of the aldosterone-sensitive distal nephron, thus increasing our understanding of how diverse renal ion transport proteins are coordinated to regulate normal blood pressure levels. Observations discussed in the present work place WNK1 and WNK4 as genes involved in the genesis of essential hypertension and as potential targets for the development of antihypertensive drugs.

Copyright ๑ 2008 S. Karger AG, Basel
\end{abstract}

Arterial hypertension is one of the most common diseases affecting more than one billion people worldwide. Most of the affected patients belong to a category known as essential hypertension, which means the origin of their disease is unknown. Unfortunately, most of the time increased blood pressure is not associated with any particular symptom, but induces a remarkable increase in the risk of fatal illnesses such as heart attack, aneurysm or stroke, due to increased pressure on the arterial wall accelerating the processes of atherosclerosis. Arterial hypertension is a prototype of what is currently known as polygenic disease, in which it is postulated that normal variation throughout the genome, due to changes at the single-base level, known as single-nucleotide polymorphisms (SNPs), predispose individuals to an increased susceptibility to environmental factors (i.e. sugar consumption and diabetes mellitus, or salt intake and arterial pressure) to produce the disease. SNPs can also be the reason for the well-known variation in the type or magnitude of response to antihypertensive drugs observed within the population (pharmacogenomics). While the origins of hypertension are unknown, several lines of evidence demonstrate that pressure natriuresis in the kidney plays a key role in the long-term regulation of arterial pressure and that arterial hypertension only occurs when the pressure natriuresis relationship in the kidney

Gerardo Gamba, MD, $\mathrm{PhD}$

Molecular Physiology Unit, Vasco de Quiroga No. 15

Tlalpan 14000, México City (México)

Tel. +52 555513 3868, Fax +52 5556550382

E-Mail gamba@biomedicas.unam.mx or gamba@quetzal.innsz.mx 
is switched to the right (guytonian hypothesis) [1]. That is, increased arterial blood pressure is required in order to achieve normal salt urinary output.

Because arterial hypertension is polygenic, in order to understand the mechanisms of the disease, it is important to identify genes involved in blood pressure regulation. A very successful approach used over the last decade has been unmasking genes causing monogenic diseases that exhibit high or low blood pressure levels under the assumption that if altered function of a single gene is enough to produce an abnormal change in blood pressure, it is highly likely that the gene could be involved in the genesis of essential hypertension. More than 15 genes have been identified following this strategy [2]. Supporting the guytonian hypothesis, all recognized genes are involved in the maintenance of renal salt balance. Good examples of genes discovered by this strategy that have increased our understanding of hypertension mechanisms are the serine/threonine kinases of the WNK family.

\section{WNK Kinases and Genetic Hypertension}

Identification of WNK Kinases as Proteins Involved in the Regulation of Arterial Blood Pressure

Two monogenic diseases resulting in abnormal variations in blood pressure exhibit mirror images in their clinical features. On the one hand, Gitelman disease (OMIM No. 263800) is an autosomal recessive disorder associated with arterial hypotension, together with hypokalemic metabolic alkalosis and hypocalciuria. This monogenic disease is due to mutations in the SLC12A3 gene located in the human chromosome 16 that encodes for the thiazide-sensitive $\mathrm{Na}^{+}: \mathrm{Cl}^{-}$cotransporter (NCC) that is expressed in the apical membrane of the distal convoluted tubule (DCT) [3-5] (table 1). The mutations are of the 'loss of function' type resulting in inactivation of the cotransporter [9-11]. The absence of NCC activity decreases salt reabsorption in the DCT, resulting in dehydration and arterial hypotension. The consequential increase in salt delivery to the connecting tubule (CNT) and collecting duct (CD) induces increased potassium and hydrogen secretion producing the hypokalemic metabolic alkalosis. On the other hand, a disease known by several names such as Gordon's disease, familial hyperkalemic hypertension or pseudohypoaldosteronism type II (PHAII; OMIM No. 145260) exhibits a mode of inheritance that is compatible with autosomal dominant transmission and features arterial hypertension that is accom-
Table 1. Genetic defects in PHAII and Gitelman disease

\begin{tabular}{lllll}
\hline Disease & Location & Gene & Defect & $\begin{array}{l}\text { Refer- } \\
\text { ence }\end{array}$ \\
\hline PHAII1 & $12 \mathrm{p} 13.3$ & PRKWNK1 & intronic deletion & 12 \\
PHAII2 & $17 \mathrm{q} 21$ & PRKWNK4 & missense mutations & 11 \\
PHAII3 & $1 \mathrm{q} 31-42$ & unknown & unknown & 11 \\
PHAII4 & unknown & unknown & unknown & 13 \\
Gitelman & $16 \mathrm{q} 13$ & SLC12A3 & mutations & $3-5$ \\
\hline
\end{tabular}

panied by hyperkalemic metabolic acidosis and hypercalciuria. This phenotype is $\mathrm{Cl}^{-}$dependent and is corrected with low doses of thiazide-type diuretics [12]. Thus, PHAII is a mirror image of Gitelman disease, strongly suggesting that increased activity of the NCC must be implicated. However, initial genomic analysis demonstrated no significant linkage of kindred with PHAII to the SLC12A3 gene on chromosome 16 in patients with PHAII [13], making it unlikely that the cause of PHAII is activating mutations of the NCC. Later, locus heterogeneity of PHAII was identified by Mansfield et al. [14] when positive linkage analysis was observed in two loci: one located in chromosome 1q31-q42 and another in chromosome 17p11-q21 (table 1). The evidence of genetic heterogeneity of PHAII was increased by Disse-Nicodeme et al. $[15,16]$, who observed in French kindreds a positive linkage to chromosome 12p13.3 and in another family no linkage to the SLC12A3 gene or to chromosomes 1, 12 and 17. Thus, at least 4 genes are capable of producing the same disease independently (table 1).

At about the time heterogeneity of PHAII was observed, in their effort to clone novel members of the mitogen-activated protein/extracellular signal-regulated protein kinases from brain tissue, the Cobb group in Texas [17] identified a new type of serine/threonine kinase that lacks the canonical lysine observed in all serine/threonine kinases in the subdomain II of the kinase domain. Due to the absence of this lysine, the new kinase was named WNK1 for 'with no lysine (K)'. This report would probably have passed unnoticed by the renal community if not for a study months later in which the Lifton group from Yale, following the positional cloning strategy [8], identified that the cause of PHAII in families with positive linkage to chromosome 12 was due to deletion of a fragment of intron 1 in the PRKWNK1 gene, encoding WNK1. 
Fig. 1. Proposed model of WNK proteins. The central kinase domain is shown by a bracket. The autoinhibitory domain and the coiled-coil domains are highlighted. Locations of the negatively charged residues in which WNK4 mutations occur are shown below WNK4.

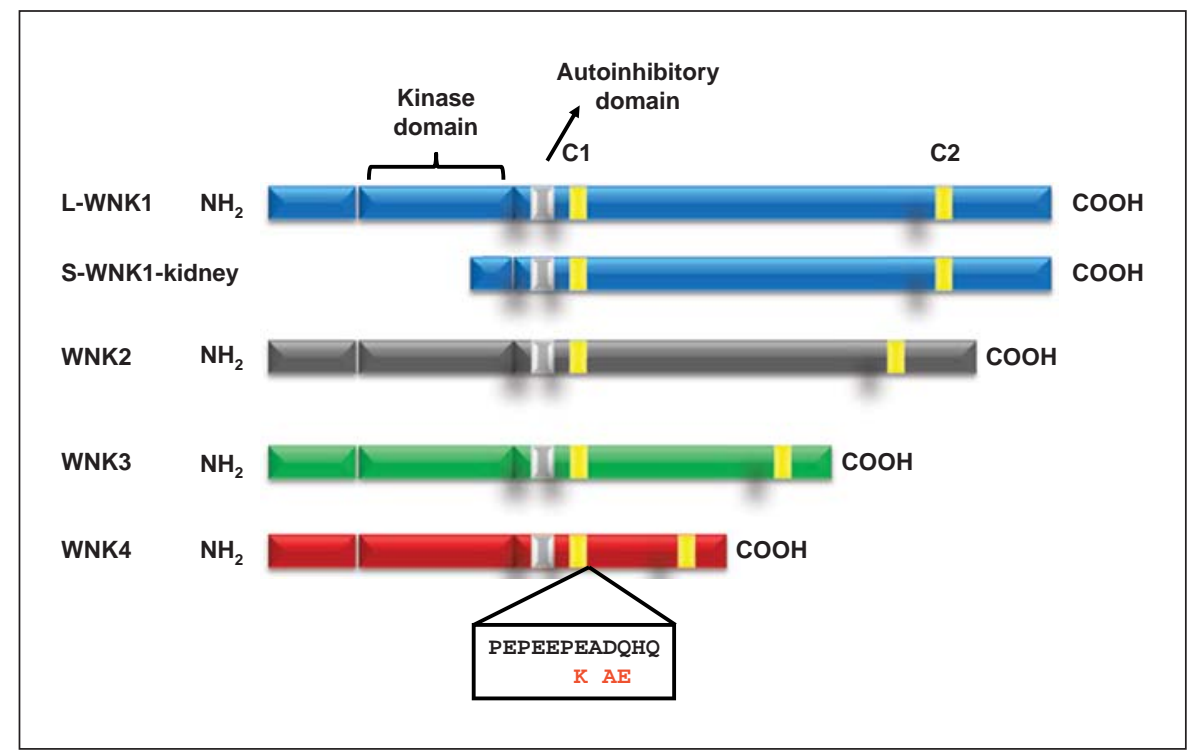

Two Forms of PHAII Are due to Mutations in WNK Kinases

Wilson et al. [8] uncovered 2 genes causing PHAII. They first observed that 2 kindreds with significant linkage to chromosome 12 p13 cosegregated with a 41 - or 21$\mathrm{kb}$ deletion, respectively, within the first large intron of $P R K W N K 1$. No mutations or deletions within the WNK1coding sequence were detected in these families. The expression of WNK1 mRNA in leukocytes in affected individuals was observed to be 5 -fold higher than in nonaffected members of the family. Thus, genomic deletions of the first intron of WNK1 increase the expression of an otherwise normal WNK1 kinase. Supporting this hypothesis, Delaloy et al. [18] later revealed that the region that goes from $-2,500$ to $-1,200$ of the human PRKWNK1 promoter represses transcription of the gene. To date, these are the only 2 PHAII kindreds in which deletion of $P R K W N K 1$ genes have been reported.

The other form of PHAII that is due to mutations in WNK kinases is the one in which positive linkage was observed with human chromosome 17. Four independent missense mutations in the PRKWNK4 gene were observed by Wilson et al. [8] in these families. Interestingly, 3 out of 4 missense mutations occur within a highly conserved acidic region that is located within the carboxylterminal domain, just after the first coiled-coil domain (fig. 1). This negatively charged region contains 10 amino acid residues that are $100 \%$ identical between $\mathrm{WNK} 1$, WNK 2 and WNK4, and 70\% with WNK3. Six out of 10 amino acid residues in this region of WNK4 are charged.
The 3 missense mutations that occur in this region change or eliminate the charge of a residue (E562K, D564A and Q565E mutations). Golbang et al. [19] later reported another PHAII kindred in which asparagine 564 is mutated to histidine (D564H). In addition, Wilson et al. [8] also observed that mutation R1185C cosegregates with PHAII in another family. This mutation, although outside the acidic domain, also changes the charge of an amino acid residue. Thus, the PHAII that cosegregates with the $P R K W N K 4$ gene is due to point mutations that change 1 charged amino acid residue. It is believed that this alteration probably affects the way in which WNK4 interacts with other proteins or charged molecules, resulting in modification of the effect of WNK4 upon transport systems.

\section{The WNK Family Is Composed of Four Members}

After the cloning and identification of WNK1 from the rat kidney [17], it was soon observed that the WNK family is composed of 4 members that were named WNK1, WNK2, WNK3 and WNK4 (fig. 1). The corresponding genes PRKWNK1, PRKWNK2, PRKWNK3 and $P R K W N K 4$ are located in human chromosomes 12, $9, \mathrm{X}$ and 17 , respectively. As shown in figure 1 , WNK kinases are composed of 3 domains. A serine/threonine kinase domain of 274 residues is flanked by a short aminoterminal domain of 146-220 amino acid residues and a large carboxyl-terminal domain of 786-1,888 residues containing an autoinhibitory domain and 2 coiled-coil domains that could be important for protein-protein in- 
teraction. The degree of identity among the $4 \mathrm{WNKs}$ at the amino- and carboxyl-terminal domains is below $20 \%$, whereas at the serine/threonine kinase it is above $80 \%$. The WNK1 kinase domain crystal structure was resolved at $1.8 \AA$ demonstrating that the catalytic lysine is located in the $\beta$-strand 2 , rather than strand 3 as in other serine/ threonine kinases [20]. Several in vitro studies have shown that WNK kinases can interact amongst each other at the protein-protein level [21-23] or by phosphorylation processes [24], as well as with many other kinase proteins including mitogen-activated protein kinases $[6,25]$, transforming growth factor $\beta$ signaling pathway kinases [26], synaptotagmin [27] and STE-20-related kinases like serine-proline-alanine-rich kinase (SPAK) or oxidative stress response kinase 1 (OSR1) [7, 28-31].

WNK1 is a ubiquitously expressed kinase with predominant expression in the kidney, heart, muscle and testis in the rat [17], mouse [32] and human [33]. In most tissues, it is predominantly expressed in polarized epithelial cells. In the kidney it is expressed along the entire nephron [32, 34]. By RT-PCR analysis it has been observed that WNK3 transcripts are present in all tissues [35], and by immunohistochemistry WNK3 protein was shown to be expressed in all nephron segments, as well as in epithelial cells of several organs such as the pancreas, biliary duct, stomach and intestine [36]. In contrast to WNK1 and WNK4, WNK3 protein is highly expressed in the central nervous system in which it is present in cell bodies of neurons expressing ionotropic $\gamma$-aminobutyric acid A receptors [36]. WNK4 is mainly expressed in the aldosterone-sensitive distal nephron. At the protein level it has been shown to be present in the DCT and CD $[8$, 34], and at the mRNA level, by single-nephron RT-PCR, WNK4 transcripts have been shown to be expressed also in the thick ascending limb of Henle's loop [34]. In addition, Kahle et al. [37] observed that WNK4 transcript and protein are present in several epithelial tissues, in which expression is more prominent in tight junctions. Interestingly, all epithelia expressing WNKs are heavily involved in $\mathrm{Cl}^{-}$transport.

The molecular diversity of the WNK family has been shown to be increased by expression of several alternative splicing variants described in WNK1 and WNK3. Two independent groups $[18,38]$ observed by Northern blot analysis $2 \mathrm{WNK} 1$ transcripts, 9.0 and $10.5 \mathrm{~kb}$ in size, that differ in the length of the $3^{\prime}$ untranslated region. These transcripts are present in all tested tissues and are known as L-WNK1. In addition, there is an approximately $8.0-\mathrm{kb}$ transcript expressed only in the kidney that is due to alternative splicing of exons $1-4$. The transcription of this isoform is under control of an intron 4 alternative promoter and contains sequences from an extra exon located between exons 4 and 5 which has been designed as exon $4 a$. As shown in figure 1, at the protein level the consequence of this splicing mechanism is a shorter WNK1 isoform that lacks the first 437 amino acid residues, including almost the entire kinase domain. This shorter, truncated isoform is known as S-WNK1. By in situ hybridization it has been demonstrated that within the kidney, L-WNK1 isoforms are present all along the entire nephron, whereas S-WNK1 is only present in the aldosterone-sensitive distal nephron, particularly abundant in the DCT and CNT. These locations are very important to understand the mechanism by which overexpression of L-WNK1 produces activation of the NCC (see below). For WNK1 another 5 different transcripts have been detected due to splicing of exons 9,11 and 12, either separately or in combination. The functional consequences of all these isoforms are unknown. Finally, human WNK3 exhibits at least 2 alternative variants. One is due to usage of an alternative splice donor into exon 18 that introduces 47 amino acid residues, and the other is due to splicing of exon 22 [35]. The functional consequences of these splicing isoforms are unknown.

\section{Modulation of Renal Ion Transport Systems by WNKs}

Several lines of evidence suggested that WNKs would be critical regulators of renal ion transport systems: (1) WNK1 and WNK4 are predominantly expressed in the aldosterone-sensitive distal nephron; (2) in addition to hypertension, PHAII patients exhibit hyperkalemia, metabolic acidosis and hypercalciuria consistent with impairment of the normal renal excretion of these ions; (3) PHAII is the mirror image of Gitelman disease that is due to inactivating mutations of the NCC; (4) the PHAII clinical picture is easily reverted by very low doses of thiazide-type diuretics [12].

\section{Effects of WNK4 upon Distal Nephron Transport \\ Systems}

Due to the mirror image between Gitelman disease and PHAII, and the exquisite sensitivity of this latter disease to thiazides, the first transport system that was studied was the NCC, which is the major salt transport pathway in the DCT (fig. 2). The effect of WNK4 upon NCC activity was assessed using the heterologous expression system of Xenopus laevis oocytes, which was used to clone these and other transporters $[39,40]$, and in which 
Fig. 2. Salt and potassium transport systems in the DCT and CNT. The DCT begins few cells after the macula densa; it is divided into 2 portions, the DCT1 in which the salt reabsorption pathway is the NCC and the DCT2 in which salt reabsorption takes place through both, the NCC and $\mathrm{ENaC}$. Then, the ENaC is the major salt reabsorption pathway in the CNT and downstream in the CD. In all these segments, the ROMK is the major potassiumsecretory pathway. As discussed in the text, L-WNK1, S-WNK1 and WNK4 are heavily expressed in these nephron segments.

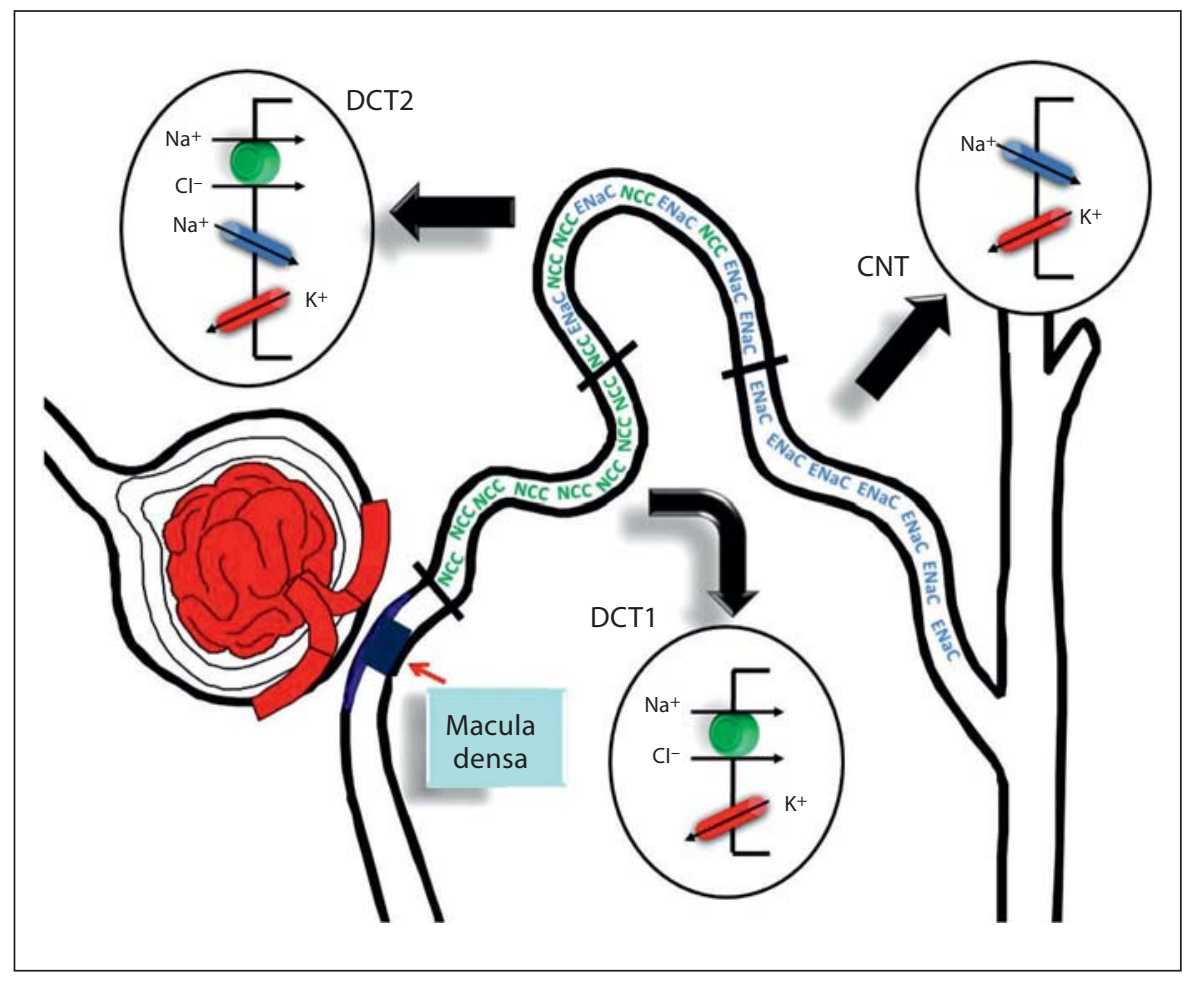

most of the functional properties of the NCC have been analyzed [41]. Two independent groups obtained comparable results simultaneously $[42,43]$. When coexpressed in oocytes, WNK4 reduces the activity of NCC by decreasing the amount of the cotransporter present on the cell surface. This negative effect upon the NCC was dependent on the WNK4 kinase activity and was also eliminated by introducing the PHAII mutation Q562E (mouse sequence; table 2). These observations were later corroborated by other groups using culture cells as expression systems $[19,44,45]$ and, also, added the evidence that decreased expression of NCC on the cell surface is not associated with increased dynamin-induced vesicle internalization but is rather due to increased lysosomal degradation of NCC protein.

PHAII is associated with hyperkalemia and potassium excretion in the nephron that predominantly occurs in the aldosterone-sensitive distal nephron. Thus, another obvious target for WNK4 regulation was the renal outer medullary potassium channel (ROMK) that is the major renal potassium-secretory pathway in the apical membrane of the distal nephron (fig. 2). Similar to NCC, WNK4 is a negative regulator of the ROMK [46]. However, there are important differences between WNK4 ef-
Table 2. Modulation of renal ion transport systems by WNKs

\begin{tabular}{lllll}
\hline & WNK4 & PHAII & S1169D & WNK1 \\
& & WNK4 & WNK4 & \\
\hline NCC & $\downarrow$ & $\uparrow$ & $\downarrow$ & - \\
ROMK & $\downarrow$ & $\downarrow \downarrow$ & $\uparrow$ & $\downarrow$ \\
ENaC & $\downarrow$ & $\uparrow$ & $\uparrow$ & $\uparrow$ \\
Claudin 4 & $\uparrow$ & $\uparrow \uparrow$ & n.d. & $\uparrow$ \\
KCCs & $\downarrow$ & $\downarrow$ & n.d. & n.d. \\
\hline
\end{tabular}

ROMK = Renal outer medullary potassium channel; $\mathrm{ENaC}=$ epithelial $\mathrm{Na}$ channel; $\mathrm{KCC}=\mathrm{K}-\mathrm{Cl}$ cotransporter; n.d. = not determined.

fects upon the NCC than upon the ROMK (table 2). First, WNK4 catalytic activity is not required for ROMK inhibition. Second, the mechanism is due to a WNK4-induced increased internalization of the ROMK via clathrin-coated pits. These observations performed originally in Xenopus oocytes have been confirmed in mammalian cells [47], in which it has been observed that WNK4 interacts with a protein known as intersectin that is in- 
volved in the clathrin-induced endocytosis. Additionally, treatment of HEK-293 cells with siRNA against WNK1 and WNK4 resulted in increased activity of the ROMK, indicating that, indeed, basal activity of the channel is modulated by both kinases [48]. Third, while for the NCC PHAII type mutations in WNK4 prevented the dominant negative effect, for ROMK they resulted in increased inhibition. This observation can explain the hyperkalemia in PHAII patients since inhibition of the ROMK is increased by mutant WNK4.

A third candidate target for WNK4 is the apical epithelial sodium channel $(\mathrm{ENaC})$ that in the distal nephron is an important pathway for sodium reabsorption (fig. 2). In addition, activity of the $\mathrm{ENaC}$ is required for ROMK potassium excretion since the luminal negative potential that is generated by sodium translocation is required for potassium to be secreted. Ring et al. [49] demonstrated that WNK4 reduces the activity of the ENaC. This effect does not require WNK4 catalytic activity and is lost by elimination of the PPXY motif of the ENaC $\beta$ - or $\gamma$-subunit, indicating that, similar to ROMK, it is due to clathrin-mediated endocytosis. Interestingly, PHAII type mutations in WNK4 prevent the inhibitory effect of WNK4 upon the $\mathrm{ENaC}$ (table 2).

The fourth identified targets for WNK4 are the proteins mediating parecellular $\mathrm{Cl}^{-}$flux in the distal nephron known as claudins. Two independent groups [50,51] observed simultaneously in MDCKII cells that WNK4 increases the paracellular transport of $\mathrm{Cl}^{-}$but not that of $\mathrm{Na}^{+}$. This effect requires WNK4 catalytic activity and is associated with phosphorylation of claudin 4 in its carboxyl-terminal domain. In addition, PHAII-type mutations in WNK4 are associated with a further increase in $\mathrm{Cl}^{-}$permeability and phosphorylation of claudin 4 (table 2). Finally, WNK4 also inhibits the activity of the K-Cl cotransporters KCC1, KCC3 and KCC4 [52], which in the distal nephron are critical for potassium secretion in the DCT [53] and acid secretion in the CD [54]. KCC4 knockout mice feature metabolic acidosis. The WNK4 inhibitory effect is kinase dependent but was not affected by PHAII-type mutations (table 2).

\section{Effects of WNK1 upon Distal Nephron Transport} Systems

The effect of WNK1 upon distal transport systems is probably more complex than WNK4 since it has been demonstrated that WNK1 is a regulator of other WNKs by phosphorylation processes [55] or by WNK-to-WNK interactions at the protein level [21, 22]. For instance, Yang et al. [43] observed that WNK1 has no direct effect upon the NCC, but prevents the WNK4-induced inhibition of the NCC. That is, when the NCC and WNK4 were coexpressed together in X. laevis oocytes, WNK4 induced inhibition of NCC activity. However, when WNK1 was added to the coexpression cocktail, the WNK4 inhibitory effect of the NCC was lost. Later on, Subramanya et al. [56] analyzed the effect of the L-WNK1 and SWNK1 variants of WNK1 upon the NCC and its WNK4 regulation. Their observations are very important to understand the mechanism by which increased expression of a normal L-WNK1 in PHAII patients produces increased activity of the NCC. These authors demonstrated that while L-WNK1 prevented the WNK4-induced inhibition of the NCC, S-WNK1 by interacting with L-WNK1 in a dominant negative fashion eliminated the L-WNK1induced inhibition of WNK4. As was discussed above, while L-WNK1 is present in several epithelial cells and along the entire nephron, S-WNK1 is mainly expressed in the DCT and CNT $[34,38]$. Thus, it is proposed that in normal subjects the ratio of S-WNK1/L-WNK1 expression in the DCT and CNT is in favor of S-WNK1, and thus, the shorter isoform by highjacking L-WNK1 prevents its inhibitory effect upon WNK4, allowing WNK4 to keep the NCC inhibited. In PHAII patients due to intronic deletions of the PRKWNK1 gene that increases expression of L-WNK1, the ratio S-WNK1/L-WNK1 is reduced. As a consequence, L-WNK1 is able to inhibit WNK4 and thus, NCC activity is increased, augmenting salt reabsorption in the DCT and thus arterial pressure. Similarly, it has been demonstrated that S-WNK1 and LWNK1 interact with each other regulating ROMK activity. Lazrak et al. [48] observed in HEK cells transfected with ROMK that L-WNK1 decreased the activity of the channel. This effect of L-WNK1 requires the L-WNK1 catalytic activity and can be prevented by coexpression of S-WNK1. In the same study it was observed that exposing rats to a $\mathrm{K}^{+}$-deficient diet was associated with increased expression of L-WNK1 and decreased expression of S-WNK1, consistent with a situation in which ROMK activity is decreased since $\mathrm{K}^{+}$secretion is maximally reduced. Consistent with these findings, O'Reilly et al. [34] also observed that the ratio S-WNK1/L-WNK1 and the expression of WNK4 at the mRNA level are modulated by low- and high-potassium diets.

WNK1 is also a modulator of $\mathrm{ENaC}$ activity. In a very interesting study, Xu et al. [57] demonstrated that ENaC can be regulated by WNK1, via another kinase known as serum glucocorticoid kinase (SGK). WNK1 induces a phosphorylation of SGK which in turn phosphorylates and inhibits a protein named Need 4 that is known to re- 
duce $\mathrm{ENaC}$ activity by promoting its endocytosis via a clathrin-dependent mechanism. Therefore, $\mathrm{ENaC}$ activity is enhanced. Interestingly, SGK is known to be a key kinase through which aldosterone achieves its effects upon distal nephron transport mechanisms. Finally, similar to WNK4, WNK1 also increases paracellular $\mathrm{Cl}^{-}$ fluxes, an effect that was associated with increased phosphorylation of claudin 4 (table 2).

\section{WNKs Lie Upstream of Kinases Involved in the}

Regulation of Ion Transporters

Biochemical and functional analysis revealed that WNK kinases and the cation-coupled chloride cotransporter (SLC12 family) interact with each other and with another family of serine/threonine kinases known as SPAK and OSR1 that belong to the STE-20 family. On the one hand, Piechota et al. $[28,58]$ observed that some members of the SLC12 family, such as the $\mathrm{K}^{+}-\mathrm{Cl}^{-}$cotransporter KCC3 and the basolateral isoform of the $\mathrm{Na}^{+}$: $\mathrm{K}^{+}: 2 \mathrm{Cl}^{-}$cotransporter, $\mathrm{NKCC} 1$, interact with SPAK in a 2-hybrid yeast system. They observed that these interactions occurred through an 'SPAK-binding domain' $[\mathrm{RFx}(\mathrm{V} / \mathrm{I})]$ present in the cotransporter sequence. Simultaneously, it was demonstrated by Dowd and Forbush [59] that modulation of NKCC1 activity by intracellular chloride concentration involved the activity of this kinase. On the other hand, Vitari et al. [29] observed that immunoprecipitation of WNK1 or WNK4 brought SPAK as an interacting protein. In this study they observed in an in vitro phosphorylation assay using the amino-terminal domain of NKCC1 that the presence of WNK1, WNK4, SPAK or OSR1 alone was not enough to achieve NKCC1 phosphorylation. Only when WNK1 or WNK4 was added together with SPAK or OSR1, did NKCC1 become phosphorylated. This effect disappeared when catalytically inactive kinases were used. The conclusion of the study was that WNK1 or WNK4 is able to induce phosphorylation of NKCC1 only when SPAK or OSR1 is present. Similar observations were obtained by Moriguchi et al. [31] for NKCC1, and also for the apical isoform of the $\mathrm{Na}^{+}: \mathrm{K}^{+}: 2 \mathrm{Cl}^{-}$cotransporter, NKCC2, and for the NCC. These observations are supported by the observations of Gagnon et al. [7] at the functional level. These authors observed in $X$. laevis oocytes that injecting WNK4 cRNA had no effect upon the NKCC1 cotransporter, unless SPAK cRNA was added to the injecting cocktail. They observed that injecting cRNA of both kinases resulted in a significant activation of NKCC1. Interestingly, this interaction does not seem to be required for WNK4-induced inhibition of the $\mathrm{K}^{+}-\mathrm{Cl}^{-}$cotransport- ers [52]. Finally, detailed definitions of the interacting domains between WNK4 and SPAK were informed $[30,60]$. Thus, it is currently believed that at least for some cotransporters, WNK1 or WNK4 lie upstream of SPAK/ OSR1 kinases.

\section{In vivo Mouse Models of PHAII}

All the above observations regarding modulation of renal ion transport systems by WNKs were done in heterologous expression systems of either amphibian or mammalian origin. Thus, it was necessary to demonstrate that the proposed mechanisms by which PAHIItype mutations in WNKs produce the disease actually occur in an in vivo model. Regarding WNK1 the only study in which genetic manipulation of WNK1 has been performed is in the WNK1 knockout mice produced by Zambrowicz et al. [61]. Mice homozygous for WNK1 deletion died before day 13 of gestation, indicating that WNK1 is an important protein for embryogenesis. However, WNK1 heterozygous mice survived to adulthood and displayed a significant reduction in arterial blood pressure, indicating that WNK1 plays a key role in maintaining normal blood pressure.

Two groups have produced transgenic mice overexpressing WNK4 containing a PHAII-type mutation. First, Lalioti et al. [62] generated bacterial artificial chromosome transgenic mice overexpressing either wild-type WNK4 or a WNK4 gene containing one of the PHAIItype mutations (Q562E). Interesting observations were done in this study: when compared with control mice, PHAII-WNK4 transgenic mice were hypertensive, whereas wild-type WNK4 transgenic mice were hypotensive. When exposed to a low- $\mathrm{K}^{+}$diet, PHAII-WNK4 transgenic mice were hyperkalemic (approx. $5.3 \mathrm{mM}$ ) and when exposed to a high- $\mathrm{K}^{+}$diet, these animals developed a dramatic increase in serum $\mathrm{K}^{+}$to values over $8.0 \mathrm{mM}$. In contrast, wild-type WNK4 transgenic mice were hypokalemic (approx. 3.7 mM). Furthermore, PHAII-WNK4 transgenic mice exhibited metabolic acidosis and were hypercalciuric. Within the histological analysis, the observations were more impressive: wild-type WNK4 transgenic mice exhibited a significant reduction in the number and size of DCTs. In contrast, PHAII-WNK4 transgenic mice exhibited a remarkable increase in the number and size of distal tubules, that is DCT hypertrophy and hyperplasia. Therefore, overexpression of PHAII-WNK4 recapitulated the PHAII phenotype, and interestingly, overexpression of wild-type WNK4 recapitulated a phenotype that is similar to Gitelman disease, in which NCC activity is absent. Finally, to find out to what extent the 
effects of WNK4 were due to modulation of NCC activity, the PHAII-WNK4 transgenic mice were crossed with NCC knockout mice. The PHAII phenotype disappeared in the new colony. Renal histology was normal, with no changes in the DCT when compared with normal controls. Arterial blood pressure and all other parameters were corrected. Even the hyperkalemia during the high$\mathrm{K}^{+}$diet was prevented, implicating NCC as a key protein, not only for the development of hypertension, but also for hyperkalemia. In the same study, treating PHAII-WNK4 transgenic mice with thiazides also corrected the phenotype. A second transgenic model that has been formed is a knockin of a PHAII mutation (D561A) that was introduced into the endogenous WNK4 locus [63]. In this study it was also observed that knockin mice reproduced the phenotype of the disease with hypertension, hyperkalemia and metabolic acidosis. At the molecular level it was shown that NCC, $\mathrm{ENaC}$ and $\mathrm{BK} \mathrm{K}^{+}$channel expression was increased, with no change in WNK4 and the ROMK. Increased phosphorylation of SPAK/OSR1 was observed in the knockin mice supporting the hypothesis that WNK4 affects the activity of NCC by interacting with, and/or phosphorylation of, STE-20 kinases. In addition, it was also observed that PHAII-WNK4 induced phosphorylation of the NCC at serine 71 where it was previously shown that its phosphorylation was associated with the activity of the NCC [64]. These studies strongly suggest that, as predicted in the heterologous expression systems, wild-type WNK4 is a potent inhibitor of NCC, and PHAII-type mutations eliminate this WNK4 property releasing the NCC from a tonic inhibition by this kinase. However, the consequences of transgenic wildtype WNK4 or PHAII-type WNK4 upon transport systems such as the ROMK, ENaC or paracelin were not reported in detail, and thus, the WNK4 in vitro effects upon this transport system still need to be confirmed in vivo.

\section{Implications of WNK4 for Renal Physiology}

The effects of WNK4 upon renal transport systems shown in table 2 reveal that WNK4 exists in at least 3 different states of function. One is the wild-type WNK4 that inhibits NCC, ROMK and $\mathrm{ENaC}$, with slight stimulation of paracellular $\mathrm{Cl}^{-}$flux. One can think of this as an equilibrium state. A second clearly identified state is the one resulting from PHAII mutations. In this state, WNK4 no longer inhibits the $\mathrm{NCC}$ and $\mathrm{ENaC}$ resulting in increased activity of these pathways, and increased inhibition of the ROMK. Simultaneously, paracellular $\mathrm{Cl}^{-}$flux is also enhanced. A third state involves the effect of WNK4 phos- phorylation by SGK kinase. WNK4 contains a canonical SGK phosphorylation site at serine 1169. Ring et al. [65] first showed that WNK4 is phosphorylated at this site by SGK. Then they observed that mutation of S1169 for alanine, which eliminates the possibility that WNK4 becomes phosphorylated at this site, had no effect upon WNK4-induced inhibition of the ROMK or ENaC. In contrast, mutation of serine 1169 for aspartic acid (S1169D) that mimics the phosphorylation state of this serine resulted in a WNK4 kinase that lost the inhibitory effect upon the $\mathrm{ENaC}$ or $\mathrm{ROMK}$, resulting in increased activity of these channels [65]. However, this mutation S1169D has no effect upon the WNK4-induced inhibition of the NCC [Gamba G., unpubl. observations]. The differential functional properties of WNK4 revealed by these different states, together with observations in PHAII patients and transgenic mice, place WNK4 as the potential kinase and the key molecular switch that explains how the kidney handles the effect of aldosterone as a salt-retaining and $\mathrm{K}^{+}$-losing hormone. In physiological conditions, losing fluid volume increases aldosterone levels, which in turn activates salt-retaining mechanisms, without affecting the normal urinary potassium excretion. This is achieved by activating the NCC, ENaC and paracellular $\mathrm{Cl}^{-}$flux, without changing or even reducing the ROMK. As discussed above, WNK4 harboring a PHAII-type mutation mimics this situation. In contrast, another stimulus for aldosterone secretion is hyperkalemia. In this situation, aldosterone release is associated with increased $\mathrm{K}^{+}$ excretion, without affecting salt retention. This is achieved by increasing $\mathrm{ENaC}$ and ROMK activity, without affecting the NCC. Thus, salt reabsorption is not increased in the DCT1 allowing enough sodium to be delivered to the DCT2 and CNT, in which the increased activity of the $\mathrm{ENaC}$ and $\mathrm{ROMK}$ promotes potassium secretion into the lumen (fig. 2).

\section{Future Research for WNKs, Renal Ion Transport and Human Hypertension}

Observations from PHAII patients, transgenic animals and in vitro transfection models together strongly suggest that WNKs and several ion transport systems in the kidney work together to maintain salt and potassium homeostasis and, as a consequence, arterial blood pressure homeostasis. Alterations in the activity of WNKs and/or transport systems are clearly associated with human diseases featuring arterial hypertension or hypotension [2]. One important area that is currently being stud- 
ied is defining the role that WNKs can have upon the most common forms of hypertension, particularly since a considerable proportion of patients with essential hypertension exhibit low plasma renin activity, suggesting that increased blood pressure in these patients is due to salt retention. In this regard, the WNK4 gene in chromosome 17 is just $1 \mathrm{Mb}$ from the locus D17S1299 that has shown the strongest linkage with hypertension in the Framingham Heart Study population [66], and recent studies suggest the association of SNPs or variants of $P R K W N K 1$ or $P R K W N K 4$ with arterial hypertension [67, 68]. It is equally important to define drug development against WNK kinases. The facts that mice heterozygous for WNK1 deletion exhibit a significantly lower blood pressure than normal mice and that PHAII-WNK4 transgenic or knockin mice are hypertensive suggest that drugs affecting the functional properties of WNK1 or WNK4 could play a potential role in the treatment of arterial hypertension.

\section{Acknowledgments}

The work performed in our laboratory has been possible thanks to the support from the Consejo Nacional de Ciencia y Tecnología (CONACYT No. 59992) and National Institutes of Health grants DK-064635 to G.G.

\section{References}

1 Guyton AC: Blood pressure control - special role of the kidneys and body fluids. Science 1991;252:1813-1816.

-2 Lifton RP, Gharavi AG, Geller DS: Molecular mechanisms of human hypertension. Cell 2001;104:545-556.

-3 Simon DB, Nelson-Williams C, Johnson-Bia M, Ellison D, Karet FE, Morey-Molina A, Vaara I, Iwata F, Cushner HM, Koolen M, Gainza FJ, Gitelman HJ, Lifton RP: Gitelman's variant of Bartter's syndrome, inherited hypokalaemic alkalosis, is caused by mutations in the thiazide-sensitive $\mathrm{Na}-\mathrm{Cl}$ cotransporter. Nat Genet 1996;12:24-30.

4 Lemmink HH, van den Heuvel LP, van Dijk HA, Merkx GF, Smilde TJ, Taschner PE, Monnens LA, Hebert SC, Knoers NV: Linkage of Gitelman syndrome to the thiazidesensitive sodium-chloride cotransporter gene with identification of mutations in Dutch families. Pediatr Nephrol 1996;10: 403-407.

5 Mastroianni N, Bettinelli A, Bianchetti M, Colussi G, de Fusco M, Sereni F, Ballabio A, Casari G: Novel molecular variants of the $\mathrm{Na}-\mathrm{Cl}$ cotransporter gene are responsible for Gitelman syndrome. Am J Hum Genet 1996; 59:1019-1026.

-6 Kunchaparty S, Palcso M, Berkman J, Zquez H, Desir GV, Bernstein P, Reilly RF, Ellison $\mathrm{DH}$ : Defective processing and expression of thiazide-sensitive $\mathrm{Na}-\mathrm{Cl}$ cotransporter as a cause of Gitelman's syndrome. Am J Physiol 1999;277:F643-F649.

7 De Jong JC, Van der Vliet WA, van den Heuvel LP, Willems PH, Knoers NV, Bindels RJ: Functional expression of mutations in the human $\mathrm{NaCl}$ cotransporter: evidence for impaired routing mechanisms in Gitelman's syndrome. J Am Soc Nephrol 2002; 13:14421448 .
8 Sabath E, Meade P, Berkman J, de los Heros P, Moreno E, Bobadilla NA, Vazquez N, Ellison DH, Gamba G: Pathophysiology of functional mutations of the thiazide-sensitive $\mathrm{Na}-\mathrm{Cl}$ cotransporter in Gitelman disease. Am J Physiol Renal Physiol 2004;287: F195-F203.

$\checkmark 9$ Mayan H, Vered I, Mouallem M, TzadokWitkon M, Pauzner R, Farfel Z: Pseudohypoaldosteronism type II: marked sensitivity to thiazides, hypercalciuria, normomagnesemia, and low bone mineral density. J Clin Endocrinol Metab 2002;87:3248-3254

10 Simon DB, Farfel Z, Ellison D, Bia M, Tucci J, Lifton RP: Examination of the thiazidesensitive $\mathrm{Na}-\mathrm{Cl}$ cotransporter as a candidate gene in Gordon's syndrome. 1995, p 632.

11 Mansfield TA, Simon DB, Farfel Z, Bia M, Tucci JR, Lebel M, Gutkin M, Vialettes B, Christofilis MA, Kauppinen-Makelin R, Mayan H, Risch N, Lifton RP: Multilocus linkage of familial hyperkalaemia and hypertension, pseudohypoaldosteronism type II, to chromosomes 1q31-42 and 17p11-q21. Nat Genet 1997;16:202-205.

12 Disse-Nicodeme S, Achard JM, Desitter I, Houot AM, Fournier A, Corvol P, Jeunemaitre X: A new locus on chromosome 12p13.3 for pseudohypoaldosteronism type II, an autosomal dominant form of hypertension. Am J Hum Genet 2000;67:302-310.

13 Disse-Nicodeme S, Desitter I, Fiquet-Kempf B, Houot AM, Stern N, Delahousse M, Potier J, Ader JL, Jeunemaitre X: Genetic heterogeneity of familial hyperkalaemic hypertension. J Hypertens 2001;19:1957-1964.

14 Xu B, English JM, Wilsbacher JL, Stippec S, Goldsmith EJ, Cobb MH: WNK1, a novel mammalian serine/threonine protein kinase lacking the catalytic lysine in subdomain II. J Biol Chem 2000;275:16795-16801.
15 Wilson FH, Disse-Nicodeme S, Choate KA, Ishikawa K, Nelson-Williams C, Desitter I, Gunel M, Milford DV, Lipkin GW, Achard JM, Feely MP, Dussol B, Berland Y, Unwin RJ, Mayan H, Simon DB, Farfel Z, Jeunemaitre X, Lifton RP: Human hypertension caused by mutations in WNK kinases. Science 2001;293:1107-1112.

16 Delaloy C, Lu J, Houot AM, Disse-Nicodeme S, Gasc JM, Corvol P, Jeunemaitre X: Multiple promoters in the WNK1 gene: one controls expression of a kidney-specific kinasedefective isoform. Mol Cell Biol 2003;23: 9208-9221.

17 Golbang AP, Murthy M, Hamad A, Liu CH, Cope G, Hoff WV, Cuthbert A, O'Shaughnessy KM: A new kindred with pseudohypoaldosteronism type II and a novel mutation $(564 \mathrm{D} \rightarrow \mathrm{H})$ in the acidic motif of the WNK4 gene. Hypertension 2005;46:295300 .

18 Min X, Lee BH, Cobb MH, Goldsmith EJ: Crystal structure of the kinase domain of WNK1, a kinase that causes a hereditary form of hypertension. Structure (Camb) 2004;12:1303-1311.

19 Yang CL, Ellison DH: WNK1 interacts physically with WNK4. J Am Soc Nephrol 2003; 14:77A

20 Yang CL, Zhu X, Wang Z, Subramanya AR, Ellison DH: Mechanisms of WNK1 and WNK4 interaction in the regulation of thiazide-sensitive $\mathrm{NaCl}$ cotransport. J Clin Invest 2005;115:1379-1387.

21 Yang CL, Zhu X, Ellison DH: The thiazidesensitive $\mathrm{Na}-\mathrm{Cl}$ cotransporter is regulated by a WNK kinase signaling complex. J Clin Invest 2007;117:3403-3411.

22 Xu BE, Min X, Stippec S, Lee BH, Goldsmith EJ, Cobb MH: Regulation of WNK1 by an autoinhibitory domain and autophosphorylation. J Biol Chem 2002;277:4845648462 . 
23 Xu BE, Stippec S, Lenertz L, Lee BH, Zhang W, Lee YK, Cobb MH: WNK1 activates ERK5 by an MEKK2/3-dependent mechanism. J Biol Chem 2004;279:7826-7831.

24 Moniz S, Verissimo F, Matos P, Brazao R, Silva E, Kotevelets L, Chastre E, Gespach C, Jordan P: Protein kinase WNK2 inhibits cell proliferation by negatively modulating the activation of MEK1/ERK1/2. Oncogene 2007;26:6071-6081.

25 Lee BH, Chen W, Stippec S, Cobb MH: Biological cross-talk between WNK1 and the transforming growth factor beta-Smad signaling pathway. J Biol Chem 2007;282: 17985-17996.

-26 Lee BH, Min X, Heise CJ, Xu BE, Chen S, Shu $\mathrm{H}$, Luby-Phelps K, Goldsmith EJ, Cobb MH: WNK1 phosphorylates synaptotagmin 2 and modulates its membrane binding. Mol Cell 2004;15:741-751.

27 Piechotta K, Lu J, Delpire E: Cation chloride cotransporters interact with the stress-related kinases Ste20-related proline-alaninerich kinase (SPAK) and oxidative stress response 1 (OSR1). J Biol Chem 2002;277: 50812-50819.

28 Gagnon KB, England R, Delpire E: Volume sensitivity of cation- $\mathrm{Cl}^{-}$cotransporters is modulated by the interaction of two kinases: Ste20-related proline-alanine-rich kinase and WNK4. Am J Physiol Cell Physiol 2006; 290:C134-C142.

-29 Vitari AC, Deak M, Morrice NA, Alessi DR: The WNK1 and WNK4 protein kinases that are mutated in Gordon's hypertension syndrome, phosphorylate and active SPAK and OSR1 protein kinases. Biochem J 2005;391: $17-24$.

-30 Vitari AC, Thastrup J, Rafiqi FH, Deak M, Morrice NA, Karlsson HK, Alessi DR: Functional interactions of the SPAK/OSR1 kinases with their upstream activator WNK1 and downstream substrate NKCC1. Biochem J 2006;397:223-231.

- 31 Moriguchi T, Urushiyama S, Hisamoto N, Iemura S, Uchida S, Natsume T, Matsumoto $\mathrm{K}$, Shibuya H: WNK1 regulates phosphorylation of cation-chloride-coupled cotransporters via the STE20-related kinases, SPAK and OSR1. J Biol Chem 2005;280:4268542693.

32 Choate KA, Kahle KT, Wilson FH, NelsonWilliams C, Lifton RP: WNK1, a kinase mutated in inherited hypertension with hyperkalemia, localizes to diverse $\mathrm{Cl}^{-}$-transporting epithelia. Proc Natl Acad Sci USA 2003; 100:663-668

- 33 Verissimo F, Jordan P: WNK kinases, a novel protein kinase subfamily in multi-cellular organisms. Oncogene 2001;20:5562-5569.

34 O'Reilly M, Marshall E, Macgillivray T, Mittal M, Xue W, Kenyon CJ, Brown RW: Dietary electrolyte-driven responses in the renal WNK kinase pathway in vivo. J Am Soc Nephrol 2006; 17:2402-2413.
35 Holden S, Cox J, Raymond FL: Cloning, genomic organization, alternative splicing and expression analysis of the human gene WNK3 (PRKWNK3). Gene 2004;335:109119.

36 Kahle KT, Rinehart J, de los Heros P, Louvi A, Meade P, Vazquez N, Hebert SC, Gamba G, Gimenez I, Lifton RP: WNK3 modulates transport of $\mathrm{Cl}^{-}$in and out of cells: implications for control of cell volume and neuronal excitability. Proc Natl Acad Sci USA 2005; 102:16783-16788.

37 Kahle KT, Gimenez I, Hassan H, Wilson FH, Wong RD, Forbush B, Aronson PS, Lifton RP: WNK4 regulates apical and basolateral $\mathrm{Cl}^{-}$flux in extrarenal epithelia. Proc Natl Acad Sci USA 2004;101:2064-2069.

38 O’Reilly M, Marshall E, Speirs HJ, Brown RW: WNK1, a gene within a novel blood pressure control pathway, tissue-specifically generates radically different isoforms with and without a kinase domain. J Am Soc Nephrol 2003; 14:2447-2456.

39 Gamba G, Saltzberg SN, Lombardi M, Miyanoshita A, Lytton J, Hediger MA, Brenner BM, Hebert SC: Primary structure and functional expression of a cDNA encoding the thiazide-sensitive, electroneutral sodiumchloride cotransporter. Proc Natl Acad Sci USA 1993;90:2749-2753.

40 Brown DD: A tribute to the Xenopus laevis oocyte and egg. J Biol Chem 2004;279:4529145299.

41 Gamba G: Molecular physiology and pathophysiology of the electroneutral cation-chloride cotransporters. Physiol Rev 2005;85: 423-493.

42 Wilson FH, Kahle KT, Sabath E, Lalioti MD, Rapson AK, Hoover RS, Hebert SC, Gamba G, Lifton RP: Molecular pathogenesis of inherited hypertension with hyperkalemia: the $\mathrm{Na}-\mathrm{Cl}$ cotransporter is inhibited by wildtype but not mutant WNK4. Proc Natl Acad Sci USA 2003;100:680-684.

43 Yang CL, Angell J, Mitchell R, Ellison DH: WNK kinases regulate thiazide-sensitive $\mathrm{Na}-\mathrm{Cl}$ cotransport. J Clin Invest 2003;111: 1039-1045.

44 Golbang AP, Cope G, Hamad A, Murthy M, Liu $\mathrm{CH}$, Cuthbert AW, O'Shaughnessy KM: Regulation of the expression of the $\mathrm{Na} / \mathrm{Cl}$ cotransporter (NCCT) by WNK4 and WNK1: evidence that accelerated dynamin-dependent endocytosis is not involved. Am J Physiol Renal Physiol 2006;291:F1369-F1376.

45 Cai H, Cebotaru V, Wang YH, Zhang XM, Cebotaru L, Guggino SE, Guggino WB: WNK4 kinase regulates surface expression of the human sodium chloride cotransporter in mammalian cells. Kidney Int 2006;69: 2162-2170

-46 Kahle KT, Wilson FH, Lifton RP: Regulation of diverse ion transport pathways by WNK4 kinase: a novel molecular switch. Trends Endocrinol Metab 2005;16:98-103.
47 He G, Wang HR, Huang SK, Huang CL: Intersectin links WNK kinases to endocytosis of ROMK1. J Clin Invest 2007;117:10781087.

48 Lazrak A, Liu Z, Huang CL: Antagonistic regulation of ROMK by long and kidneyspecific WNK1 isoforms. Proc Natl Acad Sci USA 2006; 103:1615-1620.

49 Ring AM, Cheng SX, Leng Q, Kahle KT, Rinehart J, Lalioti MD, Volkman HM, Wilson FH, Hebert SC, Lifton RP: WNK4 regulates activity of the epithelial $\mathrm{Na}^{+}$channel in vitro and in vivo. Proc Natl Acad Sci USA 2007;104:4020-4024.

50 Yamauchi K, Rai T, Kobayashi K, Sohara E, Suzuki T, Itoh T, Suda S, Hayama A, Sasaki S, Uchida S: Disease-causing mutant WNK4 increases paracellular chloride permeability and phosphorylates claudins. Proc Natl Acad Sci USA 2004;101:4690-4694.

-51 Kahle KT, MacGregor GG, Wilson FH, Van Hoek AN, Brown D, Ardito T, Kashgarian M, Giebisch G, Hebert SC, Boulpaep EL, Lifton RP: Paracellular $\mathrm{Cl}^{-}$permeability is regulated by WNK4 kinase: insight into normal physiology and hypertension. Proc Natl Acad Sci USA 2004;101:14877-14882.

52 Garzon-Muvdi T, Pacheco-Alvarez D, Gagnon KB, Vazquez N, Ponce-Coria J, Moreno E, Delpire E, Gamba G: WNK4 kinase is a negative regulator of $\mathrm{K}^{+}-\mathrm{Cl}^{-}$cotransporters. Am J Physiol Renal Physiol 2007;292:F1197F1207.

53 Amorim JB, Bailey MA, Musa-Aziz R, Giebisch G, Malnic G: Role of luminal anion and $\mathrm{pH}$ in distal tubule potassium secretion. Am J Physiol Renal Physiol 2003;284:F381F388.

54 Boettger T, Hubner CA, Maier H, Rust MB, Beck FX, Jentsch TJ: Deafness and renal tubular acidosis in mice lacking the $\mathrm{K}-\mathrm{Cl}$ cotransporter KCC4. Nature 2002;416:874878.

-55 Lenertz LY, Lee BH, Min X, Xu BE, Wedin K, Earnest S, Goldsmith EJ, Cobb MH: Properties of WNK1 and implications for other family members. J Biol Chem 2005;280: 26653-26658.

56 Subramanya AR, Yang CL, Zhu X, Ellison $\mathrm{DH}$ : Dominant-negative regulation of WNK1 by its kidney-specific kinase-defective isoform. Am J Physiol Renal Physiol 2006;290:F619-F624

57 Xu BE, Stippec S, Chu PY, Lazrak A, Li XJ, Lee BH, English JM, Ortega B, Huang CL, Cobb MH: WNK1 activates SGK1 to regulate the epithelial sodium channel. Proc Natl Acad Sci USA 2005;102:10315-10320.

58 Piechotta K, Garbarini N, England R, Delpire $\mathrm{E}$ : Characterization of the interaction of the stress kinase SPAK with the $\mathrm{Na}^{+}-\mathrm{K}^{+}-2 \mathrm{Cl}^{-}$ cotransporter in the nervous system: evidence for a scaffolding role of the kinase. J Biol Chem 2003;278:52848-52856. 
59 Dowd BF, Forbush B: PASK (proline-alanine-rich STE20-related kinase), a regulatory kinase of the $\mathrm{Na}-\mathrm{K}-\mathrm{Cl}$ cotransporter (NKCC1). J Biol Chem 2003;278:2734727353.

60 Villa F, Goebel J, Rafiqi FH, Deak M, Thastrup J, Alessi DR, van Aalten DM: Structural insights into the recognition of substrates and activators by the OSR1 kinase. EMBO Rep 2007;8:839-845.

-61 Zambrowicz BP, Abuin A, Ramirez-Solis R, Richter LJ, Piggott J, Beltrandel Rio H, Buxton EC, Edwards J, Finch RA, Friddle CJ, Gupta A, Hansen G, Hu Y, Huang W, Jaing C, Key BW Jr, Kipp P, Kohlhauff B, Ma ZQ, Markesich D, Payne R, Potter DG, Qian N, Shaw J, Schrick J, Shi ZZ, Sparks MJ, Van Sligtenhorst I, Vogel P, Walke W, Xu N, Zhu Q, Person C, Sands AT: Wnk1 kinase deficiency lowers blood pressure in mice: a genetrap screen to identify potential targets for therapeutic intervention. Proc Natl Acad Sci USA 2003;100:14109-14114.
62 Lalioti MD, Zhang J, Volkman HM, Kahle KT, Hoffmann KE, Toka HR, Nelson-Williams C, Ellison DH, Flavell R, Booth CJ, Lu Y, Geller DS, Lifton RP: Wnk4 controls blood pressure and potassium homeostasis via regulation of mass and activity of the distal convoluted tubule. Nat Genet 2006;38:11241132.

63 Yang SS, Morimoto T, Rai T, Chiga M, Sohara E, Ohno M, Uchida K, Lin SH, Moriguchi T, Shibuya H, Kondo Y, Sasaki S, Uchida S: Molecular pathogenesis of pseudohypoaldosteronism type II: generation and analysis of a Wnk4(D561A/+) knockin mouse model. Cell Metab 2007;5:331-344.

64 Pacheco-Alvarez D, San Cristobal P, Meade P, Moreno E, Vazquez N, Munoz E, Diaz A, Juarez ME, Gimenez I, Gamba G: The Na-C cotransporter is activated and phosphorylated at the amino terminal domain upon intracellular chloride depletion. J Biol Chem 2006;281:28755-28763.

65 Ring AM, Leng Q, Rinehart J, Wilson FH, Kahle KT, Hebert SC, Lifton RP: An SGK1 site in WNK4 regulates $\mathrm{Na}^{+}$channel and $\mathrm{K}^{+}$ channel activity and has implications for aldosterone signaling and $\mathrm{K}^{+}$homeostasis. Proc Natl Acad Sci USA 2007;104:40254029.
66 Levy D, De Stefano AL, Larson MG, O’Donnell CJ, Lifton RP, Gavras H, Cupples LA, Myers RH: Evidence for a gene influencing blood pressure on chromosome 17: genome scan linkage results for longitudinal blood pressure phenotypes in subjects from the Framingham Heart Study. Hypertension 2000;36:477-483.

67 Newhouse SJ, Wallace C, Dobson R, Mein C, Pembroke J, Farrall M, Clayton D, Brown M, Samani N, Dominiczak A, Connell JM, Webster J, Lathrop GM, Caulfield M, Munroe PB: Haplotypes of the WNK1 gene associate with blood pressure variation in a severely hypertensive population from the British Genetics of Hypertension Study. Hum Mol Genet 2005;14:1805-1814.

68 Tobin MD, Raleigh SM, Newhouse S, Braund P, Bodycote C, Ogleby J, Cross D, Gracey J, Hayes S, Smith T, Ridge C, Caulfield M, Sheehan NA, Munroe PB, Burton PR, Samani NJ: Association of WNK1 gene polymorphisms and haplotypes with ambulatory blood pressure in the general population. Circulation 2005;112:3423-3429. 